1

Mestre em Engenharia

e Gestão de Processos

E Sistemas (2017),

Especialista em Redes de

Telecomunicações - UFMG

(2009), com Licenciatura

Plena em Física pela

Utramig (2007) e graduação

em Engenharia Elétrica pelo

CEFET-MG (2001). Consultor

em Tecnologia e Inovação,

desenvolve projetos

relacionados à Gestão e

Segurança da Informação,

com experiência na área de

Engenharia de Processos,

ênfase em Sistemas de

Telecomunicações, Sistemas

de Comunicação de Dados,

Redes de Computadores,

Redes WiMax/LTE,

Gerenciamento de Projetos,

Engenharia de Sistemas

e Gestão de Processos

de Negócio (BPM).

2

Doutorado em Ciência da Informação na UFMG -

linha de pesquisa: Gestão

da Informação e do

Conhecimento, Arquitetura

Corporativa e Web

Semântica. Mestrado em

Administração pela FUMEC

- pesquisas na fronteira de

Sistemas de Informação,

Gestão do Conhecimento e

Administração Estratégica.

Bacharelado em Ciência

da Computação pela

FUMEC. Formação

Superior específica em

Gestão e Desenvolvimento

de Sistemas Web - XML

pelo UNI-BH. Analista de

Sistemas e programador de

computadores pela UFMG.

\section{MODELAGEM DE PROCESSOS DE NEGÓCIO APLICADA À RESTITUIÇÃO DE BENS PELA INCORPORAÇÃO DE TECNOLOGIAS DE RASTREABILIDADE.}

\author{
MODELING OF BUSINESS PROCESSES APPLIED TO THE REFUND OF \\ GOODS FOR THE INCORPORATION OF TRACEABILITY TECHNOLOGIES
}

Ricardo Antonio Bicalho ${ }^{1}$

Fernando Hadad Zaidan ${ }^{2}$

RESUMo: Considerando as tendências e os avanços tecnológicos no cenário mundial, associado ao tempo de reação nas relações comerciais entre empresas e clientes, é importante que as organizações estejam com suas estruturas administrativas preparadas para disponibilizar serviços de interação rápidos, confiáveis e seguros. No ambiente da Segurança Pública, o objetivo central deste artigo foi identificar por meio da operacionalização das técnicas de modelagem de processos de negócio (BPMN), que incorporadas às demandas das organizações, diminua o tempo de resposta para as ações necessárias referentes à restituição de bens apreendidos derivados de crimes contra o patrimônio, extraviados em deslocamento ou de procedência duvidosa. Desta forma, como resultado, foi possível identificar e compreender os aspectos do comportamento organizacional e interorganizacional das instituições. A expectativa foi estabelecer parâmetros que poderão sensibilizar sobre a necessidade da criação de novos procedimentos, para o aumento da eficiência e a inclusão da tecnologia, principalmente em processos jurídicos que envolvem bens apreendidos e a política criminal no Brasil.

Palavras-chave: Morosidade Processual; Destinação de bens apreendidos; Gestão de Processos de Negócio; Gestão de bens apreendidos; Rastreabilidade.

ABSTRACT: Considering the trends and technological advances in the world scenario, coupled with the reaction time in business relations between companies and customers, it is important that organizations have their administrative structure prepared to provide fast, reliable and secure interaction services. In the Public Security environment, the main objective of this article was to identify, through the operationalization of business process modeling techniques (BPMN), which are incorporated into the demands of the organizations, reducing the response time for the necessary actions related to the restitution of goods seized from crimes against property, lost in displacement or of dubious origin. As a result, it was possible to identify and understand the aspects of the organizational and interorganizational behavior among institutions, as well as to establish parameters that could raise awareness of the need to create new procedures, increase efficiency and include technology, especially in legal proceedings involving seized assets and criminal policy in Brazil.

KEYWORDs: Procedural delays; Allocation of seized goods; Business Process Management; Management of seized assets; Traceability. 


\section{INTRODUÇÃO}

Desde o início do século XXI, o cenário jurídico mundial vem sofrendo transformações no que se refere à necessidade de modernização dos processos penais acerca dos procedimentos aplicados na gestão de bens apreendidos. Em países como a França, Itália e Portugal, diversos projetos foram desenvolvidos no sentido de se criar soluções para apoiar o Poder Judiciário, na administração recursos confiscados de atividades ilícitas. No Brasil, o Ministério da Justiça (MJ), apoiado pelo Conselho Nacional de Justiça (CNJ), iniciou em 2003 diversas ações que materializaram o Sistema Nacional de Bens Apreendidos em 2008 e a consolidação do "Manual de Bens Apreendidos" em 2011 (JUSTIÇA, 2011).

Para auxiliar no controle de bens apreendidos, as possibilidades de gestão ou de rastreabilidade ficam condicionadas a procedimentos documentais que apresentam baixo resultado de resposta em relação às demandas de entrada e saída. Para o caso de processos jurídicos que envolvem crimes contra o patrimônio, a capacidade de restituição de bens patrimoniais (ativos) ao indivíduo apresenta-se muito lenta e dentro de uma visão moderna existem equipamentos e sistemas disponíveis no mercado que podem auxiliar no processo. Surge então como alternativa a aplicação da tecnologia Radio Frequency Identificator (RFID) que se refere a um sistema automatizado para controle e monitoramento de indicadores, que pode apresentar resultados relevantes para o planejamento e gestão do conhecimento para todos os ativos incorporados ao sistema (ROBERTO et al., 2013).

A gestão dos ambientes onde estão armazenados os ativos custodiados pelo poder judiciário, assim como novos bens incluídos nos sistemas por empresas ou instituições privadas, aumenta a capacidade de rastreabilidade, identificação e restituição seja por extravio, furto, roubo, desvio condicional ou de origem desconhecida. Segundo Comploier (2013), haveria novas expectativas para atuação do Estado em relação à privação dos ganhos decorrentes de atividades ilícitas, por cooperação jurídica entre órgãos de segurança no Brasil e pelo desenvolvimento de sistemas normativos mais eficientes. $\mathrm{O}$ objetivo deste estudo foi analisar a possibilidade da diminuição do tempo de resposta na administração e restituição de bens apreendidos, derivados de crimes contra o patrimônio, extraviados ou de procedência duvidosa por meio da operacionalização das técnicas de modelagem de processos de negócio (BPMN).

Práticas de gestão, com o foco em métodos relacionados à inovação tecnológica compartilhada com tendência aberta, e a parametrização dos múltiplos modelos organizacionais públicos e privados pela aplicação da Gestão de Processos de Negócio (Business Process Management - BPM), poderão conduzir a uma reflexão crítica sobre os procedimentos mais adequados nas essências das cadeias de valores e suas relações. As aplicações de iniciativas BPM podem apoiar o alinha- 
mento entre as áreas estratégicas e operacionais, de modo que aumente a capacidade de resposta das instituições através de um conjunto de técnicas para melhoria contínua (SANTOS; SANTANA; ALVES, 2012).

A proposição de um modelo mais adequado relacionado ao contexto de gestão de processos dentro das organizações, será importante quanto aos parâmetros de decisão necessários para o ajuste da logística do fluxo e controle de materiais sob custódia pública ou privada. $\mathrm{Na}$ gestão de processos de negócio, as tarefas estão vinculadas a um ciclo de vida que compreende o planejamento, aquisição, incorporação, administração e desincorporação do recurso (ABPMP, 2013). A rastreabilidade através da aplicação de recursos tecnológicos e a interoperabilidade também poderá viabilizar a restituição rápida dos bens patrimoniais, sejam eles envolvidos ou não em processos penais. A estrutura desse artigo foi desenvolvida em cinco capítulos destacando:

I. introdução, estrutura do trabalho e os objetivos a serem alcançados;

II. metodologia de pesquisa aplicada;

III. revisão da literatura destacando os principais conceitos;

IV. apresentação da pesquisa e realização da discussão dos resultados;

V. considerações finais, as limitações do estudo e as sugestões de continuidade.

\section{METODOLOGIA}

Escolheu-se um método aplicado no intuito de realizar uma pesquisa e, em seguida, mapear e analisar os processos identificando a relação e o comportamento das organizações entre os diversos níveis existentes. A partir da comparação e a interação entre pontos comuns, em relação aos processos identificados, foi possível mapear os parâmetros mais adequados para análise, que permitiram um ajuste de sensibilidade relevante para obter os melhores resultados.

A aplicação de técnicas derivadas do BPMN viabilizou uma base para o desenvolvimento desta pesquisa através do gerenciamento de processos de negócios, estabelecendo uma cadeia de valores que permita melhorar os índices de satisfação dos serviços associados. Por meio de indicadores visuais, a notação ofereceu os recursos que permitiram uma automação das atividades de forma simples e eficaz, gerando assim um modelo de fluxograma que atende tanto as necessidades para o aumento da eficácia quanto da eficiência dos sistemas e recursos aplicados.

\section{REVISÃO DE LITERATURA}

Este capítulo descreve os principais fundamentos que serão importantes para subsidiar o desenvolvimento desta pesquisa sob o contexto jurídico, administrativo organizacional e tecnológico. Foram 
considerados também aspectos relacionados aos procedimentos ligados aos processos jurídicos que envolvem a política criminal no Brasil e a custódia dos bens apreendidos. Procedimentos e a aplicação de ferramentas e técnicas de Gestão de Processos de negócio (BPM) tornam-se cada vez mais necessárias, para que através da intervenção tecnológica, seja possível realizar uma administração e gestão mais eficiente.

\section{RECUPERAÇÃO E CUSTÓDIA DE BENS APREENDIDOS}

As atividades relacionadas aos materiais ou artefatos que são agrupados em função de terem origem desconhecida, possuem um conjunto de regras e procedimentos que possibilitem o arquivamento e registro dentro das instituições constitucionalmente responsáveis.

Métodos relacionados à apreensão, administração e destinação de bens acautelados pela Justiça criminal, temas deixados em segundo plano dentro do processo penal, começam a necessitar de uma visão de modernização da arquitetura dos processos através de estudos mais aprofundados (COMPLOIER, 2015). Neste contexto, a responsabilidade de realizar a manutenção e conservação dos bens acautelados passa a ser do Estado que processa essa gestão de todo conjunto patrimonial. Este processo se torna importante para duas situações: 1) um investigado pode se tornar um réu absolvido, tendo seus bens restituídos em boas condições ou 2) tornar um réu condenado onde, neste caso, os bens seriam levados a leilão com um baixo grau de depreciação.

Ainda segundo Comploier (2015), o processo de gestão se apresenta muito importante quando da aplicação da alienação antecipada, ainda pouco aplicada pelo Sistema Judiciário, em que se preserva o valor dos bens acautelados. O Estado se torna um destinatário imediato para casos em que o réu é condenado e, quando há absolvição, ocorrerá a recuperação do valor dos bens que foram apreendidos. Iniciativas internacionais também foram desenvolvidas com a finalidade de processar um modelo para a gestão de bens apreendidos completo e mais eficiente, que pudesse apoiar uma reação positiva devido à morosidade para as decisões judiciais que refletia na descapitalização destes bens.

Desta forma, no mapeamento dos processos de recuperação e custódia, com a identificação de todos os níveis e atividades envolvidas nesta arquitetura, será possível visualizar protótipos que realize a administração de todo o sistema em favor do Estado. O estabelecimento de um modelo de interoperabilidade para gestão poderá contribuir em um melhor tempo de resposta, na interação entre o Poder Judiciário, instituições de segurança pública e os indivíduos envolvidos diretamente e indiretamente nos processos penais. No escopo dos diversos projetos que estão ligados à administração de bens apreendidos, surgem oportunidades de negócio que podem ser apoiadas pela iniciativa privada, inclusive pela aplicação de tecnologias emergentes para rastreio de documentos e objetos (COUTINHO et al., 2014). 


\section{PROCESSOS JURÍDICOS E A POLÍTICA CRIMINAL NO BRASIL}

A identificação de recursos tecnológicos que sejam aplicáveis dentro das normas jurídicas podem apoiar ações no sentido de combater a propagação dos crimes ligados ao patrimônio. A partir da combinação de todas as informações com processos de monitoramento poderão ser viabilizadas formas de gerenciamento mais adequado de toda a cadeia documental para os bens que são custodiados e gerenciados pelo Estado. O fluxo de informações tende a ser bidirecional, iniciando em sistemas gerenciais para documentos (fluxo de gravação) e destes para sistemas gerenciais (fluxo de leitura) no qual um conjunto de sistemas apoia na gestão da inércia destas informações e de objetos associados distribuídos ao longo da cadeia (PEDROSO; ZWICKER; SOUZA, 2009).

Relatórios trimestrais da Ouvidoria do Conselho Nacional de Justiça, referente ao último trimestre de 2015 e primeiro trimestre de 2016, apontam um crescimento de $22 \%$ no número de reclamações em função da morosidade processual no Poder Judiciário, fato que pode refletir no número de bens custodiados e armazenados nos estados (NACIONAL, 2016).

\section{INSTITUIÇÕES DE SEGURANÇA E DO PODER JUDICIÁRIO}

A política criminal no Brasil envolve aspectos constitucionais que hierarquizam as organizações e definem as responsabilidades dentro do estado democrático de direito. O modelo topológico que envolve as estruturas administrativas na segurança pública, começa com agentes públicos, que recebem investimentos para combater organizações criminosas totalmente desproporcionais à movimentação financeira de atividades ilícitas. Desta forma, a ineficiente e a falta efetiva de gestão dos bens apreendidos, sequestrados, arrestados, bloqueados e determinados perdidos pelo sistema de Justiça Penal não consegue reverter rapidamente recursos para subsidiar o Estado no combate ao crime (COMPLOIER, 2015).

Um novo modelo de inteligência administrativa pode apoiar todas as organizações do poder público que almejam uma evolução estratégica e integrada das esferas estaduais e federais. $\mathrm{O}$ desafio de gerenciar com efetividade, ativos apreendidos e confiscados nos processos judiciais, perpassa em identificar e analisar os problemas estatais para gerenciamento de ativos apreendidos ou confiscados (SAADI; MACHADO, 2015). A atualização dos processos administrativos e a inovação dos métodos de cadastro de bens apreendidos vinculados a procedimentos criminais poderá permitir maior rastreabilidade destes objetos.

\section{PROCEDIMENTOS E TÉCNICAS DE GESTÃO}

Procedimentos jurídicos associados à gestão de processos que envolvem a investigação patrimonial, administração e destinação de bens apreendidos e confiscados ou perdidos estabelecem uma série de 
cenários que se torna conveniente analisar os níveis de interação e os resultados das atividades vinculadas. Entretanto, dentro da investigação criminal no Brasil, as ações estão voltadas à apreensão de objetos necessários à prova de um delito ou infração, incluindo também coisas de baixo valor econômico (TINOCO; CHAHINE, 2012).

No direito brasileiro, a destinação provisória de bens ocorre como alienação antecipada ou administração judicial que se subdivide em três seguimentos: a) o encaminhamento para depósitos judiciais ou policiais; b) a nomeação de um depositário ou administrador; c) a destinação dos bens para uso provisório de entidades públicas ou com finalidade social (COMPLOIER, 2015). Segundo Critsinelis (2013), em uma gestão inteligente do patrimônio privado confiscado em um processo criminal, é importante considerar a classificação dos bens em função da abrangência de propriedades, pessoas físicas ou jurídicas, e a origem da apreensão. De um modo geral, a destinação de bens no direito brasileiro baseado no Código de Processo Penal (CPP), antes de transitar em julgado a sentença final, não poderão ser restituídos enquanto interessarem ao processo (art. 118, CPP).

No sentido de apoiar estes procedimentos, foi criado em 2008, pelo Conselho Nacional de Justiça (CNJ), o Sistema Nacional de Bens Apreendidos (SNBA) para dimensionar os mecanismos de suporte e gestão de bens patrimoniais contidos nos processos penais (LIMA, 2012). Desta forma, para operacionalizar e tornarem mais claros os procedimentos da custódia e destino dos ativos patrimoniais, o CNJ, portanto, estabeleceu em 2011 o Manual de Bens Apreendidos como uma ferramenta em busca de uma justiça mais adequada no Brasil contemporâneo. O SNBA é um sistema que consolida um banco de dados único que possui informações de bens apreendidos em processos criminais em todo o Brasil (JUSTIÇA, 2011).

\section{MODELOS E TÉCNICAS DE GESTÃO DE PROCESSOS DE NEGÓCIO}

Para se realizar a operacionalização das técnicas e conceitos relacionados à gestão dos processos, é importante o início da abordagem através do Gerenciamento de Processos de Negócio (BPM), sendo possível proporcionar o alinhamento de visão entre as áreas estratégicas e as funcionais envolvidas. Isso irá permitir uma harmonia quanto o aumento da capacidade das organizações, de modo à prepara-las para responder às mudanças e inovações em um mercado muito competitivo (SANTOS; SANTANA; ALVES, 2012).

A primeira etapa do modelo está relacionada a uma visão do cenário atual (AS-IS) na qual é possível realizar a diagramação de todos os parâmetros, níveis e camadas existentes nos fluxos e processos já estabelecidos. A análise envolve a compreensão de processos de negócio, considerando ainda sua eficiência e eficácia para alcançar os objetivos identificados. Na compreensão dos processos atuais é importante a sua decomposição, aplicação de técnicas analíticas, análise de escopo, contexto de negócio, regras e métricas de desempenho (ABPMP, 2013). 
No segundo momento, é importante a idealização de um modelo futuro de processo de negócio (TO-BE), em que há preocupação com objetivos do negócio e de desempenho de processos, pois desta forma, é possível verificar planos e diretrizes sobre como fluxos de trabalho, aplicações de negócio, plataformas tecnológicas, recursos de dados, controles financeiros e operacionais interagem com os processos. Associando o modelo de Gestão de Processo de Negócios, para mapeamento dos processos iniciais, com as tecnologias de rastreabilidade e interação existentes no mercado, será possível através de um modelo futuro, estabelecer parâmetros de fluxo e mais eficientes.

\section{TECNOLOGIAS ASSOCIADAS À GESTÃO}

Em diversas áreas das empresas e instituições são aplicadas infraestruturas de hardwares e softwares que têm como objetivo aumentar a eficiência e a gestão dos processos. A implementação de tecnologias de rastreabilidade através de códigos de barras nas operações, por exemplo, pode consideravelmente aumentar a qualidade dos serviços para diminuir o tempo de coordenação e processamento das ações (MODRAK; KNUTH, 2012).

Considerando complementar as soluções atualmente utilizadas, existem tendências de aplicação da Radio Frequency Identificator (RFID) e Near Field Communication (NFC), associadas também à tecnologias progressivas, como interfaces de serviços através da internet, que possibilita a obtenção de um sistema automatizado para gerenciamento, com oportunidades no campo da segurança, responsabilidade e rastreabilidade de documentos e objetos (MODRAK; KNUTH, 2012).

Pode-se identificar, portanto, que existem diversas formas de realizar uma gestão de processos através da aplicação de tecnologias de rastreabilidade. A diagramação das camadas de serviço e dimensionamento do fluxo das atividades irá permitir identificar, pela aplicação de tecnologias, o mapeamento e monitoramento contínuo de processos visando aumentar a capacidade de organização e a eficiência em todos os segmentos.

\section{GOVERNANÇA E POLÍTICAS PÚBLICAS}

Algumas Instituições do cenário público nacional apresentam mecanismos de gestão que são importantes para a administração e que contribuem como apoio para os processos de decisão. Entretanto, mesmo com a disponibilidade de ferramentas de integração, os resultados da interação entre diversos níveis da administração ainda precisam ser modernizados.

A governança eletrônica em instituições públicas é importante para que os processos de gestão tenham subsidio estratégico no sentido de aumentar a eficiência entre os elos da administração. O surgimento de novas estruturas de Tecnologia da Informação e Comunicação (TIC) pode apoiar o desenvolvimento de projetos, oferecendo arranjos for- 
mais e informais que determinam como serão as ações políticas futuras frente aos problemas de gestão e do ambiente que está em constante mudança (BRAGA et al., 2008).

Em um cenário em que considera-se a necessidade de investimentos, o estabelecimento de parcerias poderia apoiar mais rapidamente a modernização e ampliação das facilidades de interação entre o governo e o cidadão. Um dos aspectos que pode limitar a governabilidade está associado às condições de deterioração dos ambientes sociais, o que limita os investimentos dos governos em todos os setores.

Diante da dificuldade das instituições políticas e administrativas em tratar os desafios pela crescente complexidade, a governança surge como um processo interativo para resolver problemas (FREY, 2000). Dentro deste contexto, a aplicação de Parcerias Público Privadas (PPP) pode ser uma alternativa para acelerar a modernização e a inovação no Estado apoiando também o desenvolvimento de interfaces mais rápidas e eficientes com o cidadão (CARRARA, 2008).

Em uma situação em que o cidadão busca por serviços para o registro de ocorrências de furto, roubo ou extravio de objetos, há o início de um processo de interação com sistemas, que ainda não apresenta uma inteligência sistêmica rápida e eficiente para a restituição do artefato em caso de ser localizado. Neste contexto, a implantação de tecnologias de rastreabilidade para a gestão de bens apreendidos, em parceira com o setor privado (PPP), poderia ser relevante no sentido racionalizar os tempos de interação, e estruturando ações de governança que configurem a satisfação do cidadão (MEDEIROS; GUIMARÃES, 2005).

\section{APRESENTAÇÃO DE PESQUISA}

Para que seja possível um melhor resultado da incorporação de tecnologias de rastreabilidade, como apoio para a administração de bens apreendidos, é importante avaliar todos os recursos humanos e de infraestrutura disponíveis, o que será mostrado nos modelos que seguem.

\section{MODELAGEM DO CENÁRIO ATUAL}

Os cenários em que ocorrem os procedimentos de recepção e arquivamento dos objetos apreendidos ou recuperados foram analisados a partir de amostras não probabilísticas por acessibilidade em diversos pontos de concentração de recebimento e processamento de ocorrências. O diagnóstico dos procedimentos para o dimensionamento e modelagem do cenário atual (AS-IS) foi realizado inicialmente por meio de visita às unidades que recebem e realizam o registro das ocorrências. O universo da pesquisa considerou o estado de Minas Gerais (MG), em 10 unidades no município de Belo Horizonte e 5 unidades em Contagem, para um total de 40 questionários que procurou avaliar o comportamento, o fluxo de atividades destas infraestruturas e os 
processos associados, foi identificado que a interação entre os recursos ocorre de maneira sistêmica

\section{GESTÃO DAS OCORRÊNCIAS}

Como mecanismo de assistência e monitoramento são utilizadas ferramentas computacionais para garantir o acompanhamento dos Registros dos Eventos de Defesa Social (REDS) com o objetivo de apoiar o Estado de MG no estabelecimento de estratégias e ações de combate à criminalidade.

O REDS é um grande sistema de gestão de ocorrências aplicado em MG, que recebe o registro de qualquer ilícito penal envolvendo o cidadão e objetos que façam parte ou não de um ato criminoso. A inclusão destes registros pode ocorrer pela ação de agentes públicos, através de processos e etapas de validação, que qualifica e tipifica o ato infracional com a identificação de seus autores associado às provas da pratica delituosa.

Para o caso dos objetos recolhidos/apreendidos em 2016, que representou 9,89\% em relação a todas as ocorrências lançadas nos Registros de Eventos de Defesa Social (REDS), uma maior agilidade nos procedimentos de encaminhamento para a justiça poderia acelerar os processos em tal ponto que facilitaria uma alienação antecipada destes artefatos em favor do Estado. Essa medida, respeitando as regras de destinação de bens no direito brasileiro baseado no Código de Processo Penal (CPP), viabilizaria investimentos em segurança pública pela conversão monetária dos bens apreendidos. Em 2011, apenas 0,23\% foi objeto de alienação antecipada, ação que pode ser uma alternativa viável em relação aos bens apreendidos (LIMA, 2012).

\section{NÍVEIS DE PROCESSAMENTO DAS OCORRÊNCIAS}

Uma primeira ação de acesso aos sistemas acontece quando o cidadão deseja realizar o registro de uma ocorrência de furto, roubo ou extravio de objetos e se desloca a um ponto de presença dos agentes públicos de segurança para notificar o fato ocorrido. Neste momento, surge o primeiro nível de interação que consiste em identificar os envolvidos e o relato circunstancial que será utilizado para apuração dos fatos através dos recursos físicos e humanos disponíveis em cada ponto de presença (CARRIJO; ALVARENGA, 2011). A análise dos questionários aplicados permitiu identificar que, embora tenha predominado o acesso em apenas um sistema, apenas um recurso humano é utilizado para realizar o atendimento presencial para o registro efetivo da ocorrência.

Outro tipo de processamento ocorre quando há apreensões ou operações que resultem no confisco de bens derivados de ações ou de práticas criminosas que também são analisados e investigados. Em específico, para o caso dos artefatos extraviados, esta pesquisa considera o processamento da ocorrência, sendo realizada de modo virtual pela 
própria vítima através da interação com sistemas via web, identificado inicialmente pela notação BPMN conforme a Figura 1.

FIgURA 1 - Níveis de Processamento

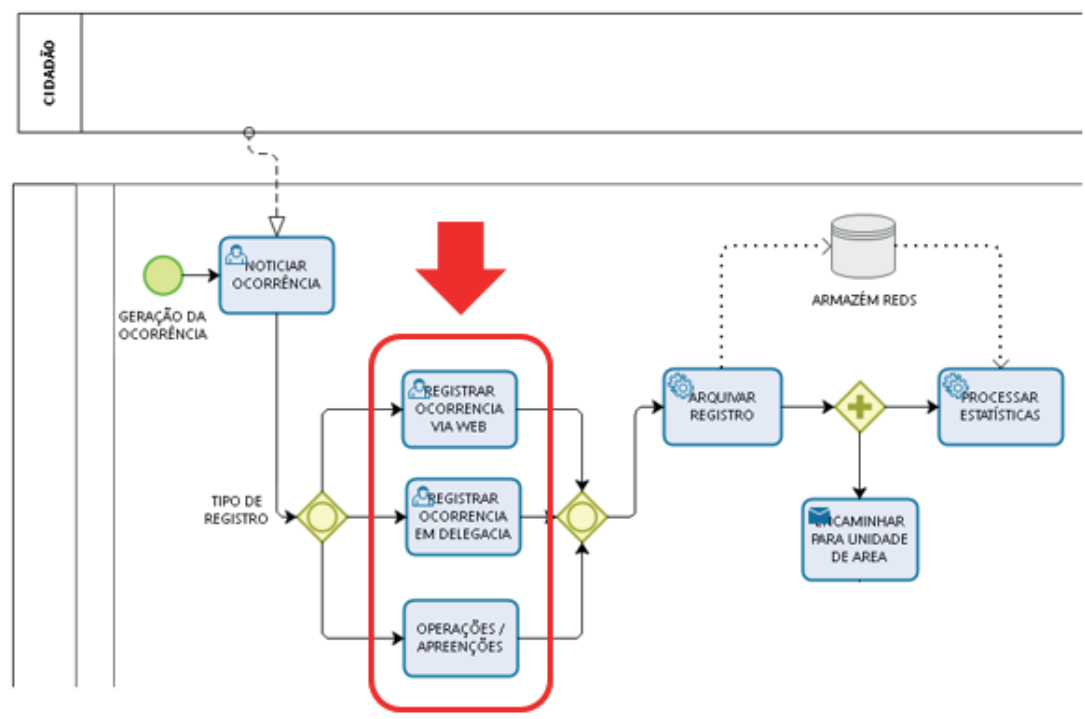

FONTE: Elaborado pelo autor, 2017.

Para o registro via web não há necessidade de deslocamento do cidadão a um ponto de presença, pois todos os procedimentos são realizados pela internet e o fluxo de apuração ocorrerá normalmente a partir do encaminhamento para a unidade de área.

\section{REGISTRO DE OCORRÊNCIAS POR FURTO/ ROUBO E APREENSÕES DE OBJETOS}

Todas as ações de registro das ocorrências são coordenadas e gerenciadas pelos operadores de segurança pública, através de equipes específicas e especializadas que executam protocolos no sentido de garantir os direitos do cidadão em ter seus bens recuperados. $\mathrm{O}$ atendimento ocorre pela ação da vítima em registrar o fato, onde, a partir daí, haverá um tempo de atendimento relacionado ao cadastro e processamento da informação apresentada.

Considerando todos "pontos de presença" de segurança pública existentes em MG para o atendimento e recebimento de ocorrências, foi realizado um mapeamento dos processos, a fim de identificar os procedimentos e recursos utilizados na ação destes registros. Entre os níveis de interação nestes pontos, verificou-se a aplicação de recursos humanos gerenciados e orientadas para, além de realizar os registros, promover tentativas de localização de objetos roubados/furtados até a sua restituição, ou o encaminhamento dos artefatos apreendidos para a Justiça.

Analisando os dados extraídos dos questionários aplicados para avaliar o comportamento, o fluxo de atividades destas infraestruturas e os processos associados, foi identificado que a interação entre os recursos ocorre de maneira sistêmica. Através da observação do compor- 
tamento organizacional foi possível visualizar o envolvimento mínimo de um agente público de segurança para o registro de uma ocorrência com natureza de roubo/furto através do acesso de 1 a 2 sistemas, com o mínimo de cinco procedimentos para finalizar o registro.

Por meio do BPMN, destaca-se inicialmente um tempo de atendimento ao cidadão que variou de 20 a 30 minutos, provocando a geração de um registro estático modelado conforme a Figura 2. Posteriormente, são designadas equipes especializadas para executarem protocolos, no sentido de se tentar localizar os objetos furtados/roubados descritos nas ocorrências.

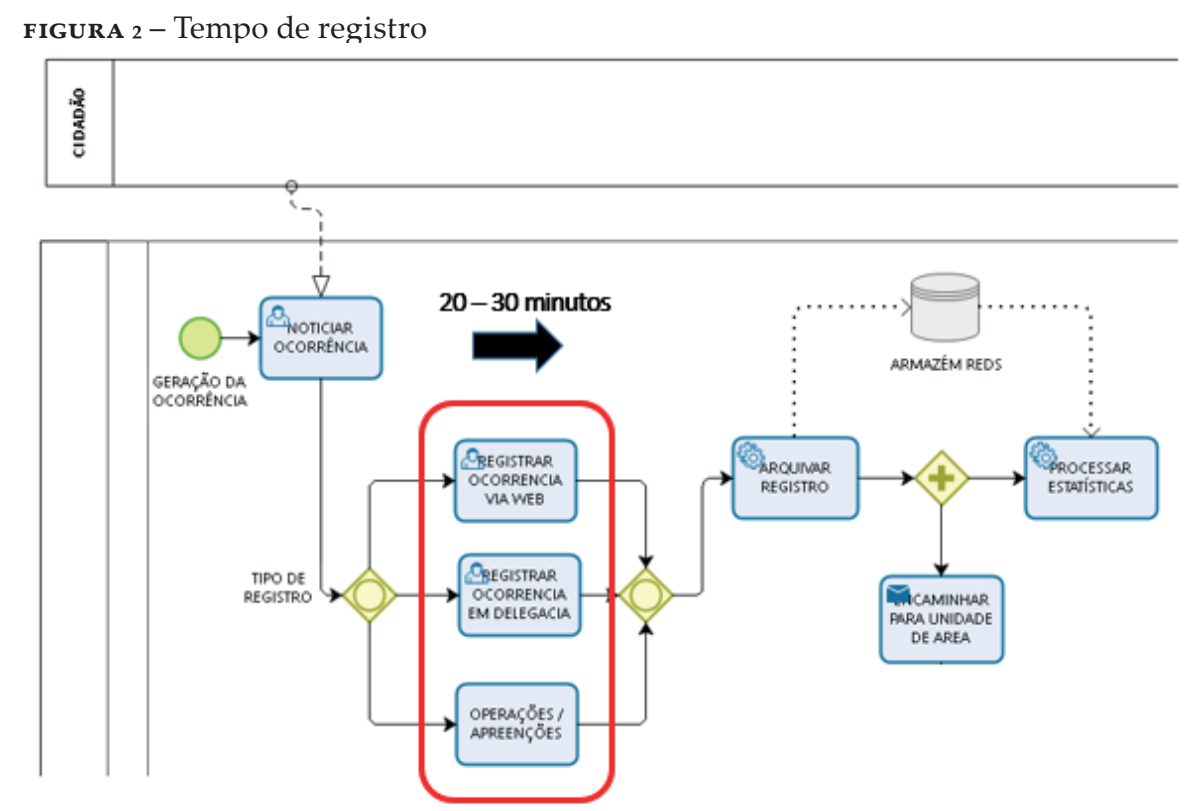

FONTE: Elaborado pelo autor, 2017.

Entretanto, após a geração deste registro, não existem métricas que permitam estimar qual é o tempo de espera para o início das ações de localização, o que atrasa a expectativa de resposta para a vítima e reflete um primeiro nível de ineficiência de reação das instituições de segurança. Ainda nesta etapa, todas as informações relatadas são inseridas em um banco de dados REDS que não possui integração direta com bancos externos ou mecanismos de interação tecnológicos que viabilizem um primeiro diagnóstico de localização.

Para o caso em que os objetos derivados de ações ilícitas são apreendidos, o fluxo de processamento envolve equipes que documentam as ações e encaminham todos os artefatos para armazenamento. Neste cenário de 2 a 5 recursos humanos em atividade no "ponto de presença", formalizam o registro da ação ocorrida nas operações, realizando um mínimo de 6 etapas para a identificação do material e o registro documental e sistêmico da apreensão conforme Figura 3. 


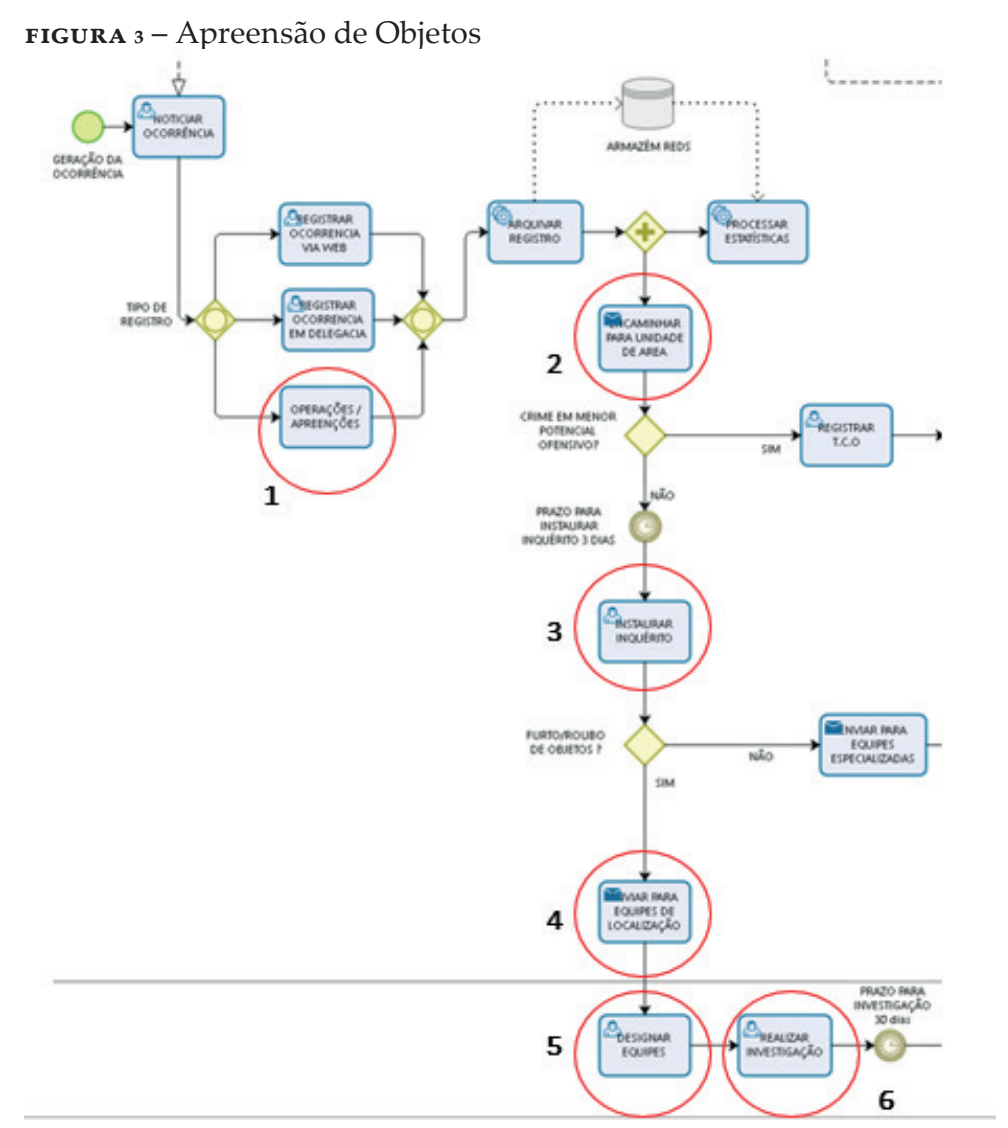

FONTE: Elaborado pelo autor, 2017.

Diante dos resultados obtidos dos questionários aplicados, observou-se também que mais de dois sistemas são acessados onde, a administração e rastreabilidade dos objetos apreendidos são realizadas através de etiquetas em papel com o encaminhamento posterior para uma área de armazenamento.

De acordo com a Figura 3, observa-se que o início das ações de investigação do objeto apreendido ou relatado como furtado/roubado só ocorre na etapa 6 com o uso de recursos humanos e ferramentas computacionais específicos, aplicados para cada tipo ou natureza dos objetos. Isso significa que para o caso de um aparelho celular, existe a possibilidade de interação com as redes das operadoras de telefonia, mas, que para o caso de uma bicicleta, há uma limitação tecnológica para efetuar um rastreamento eficiente.

Na ação de apreensão de objetos, o modelo (AS-IS) em destaque, conforme a Figura 4, retrata parte do processo que utiliza o PCNET para formalizar a ocorrência, mas não há interação automática entre a apreensão e o encaminhamento para o Poder Judiciário, no qual o registro documental de envio dos objetos é realizado via ofício. Os resultados da aplicação dos questionários demonstraram que os tempos variaram entre 30 e 180 dias, podendo ocorrer situações em que demore mais de um ano até que os objetos sejam remetidos à justiça. 


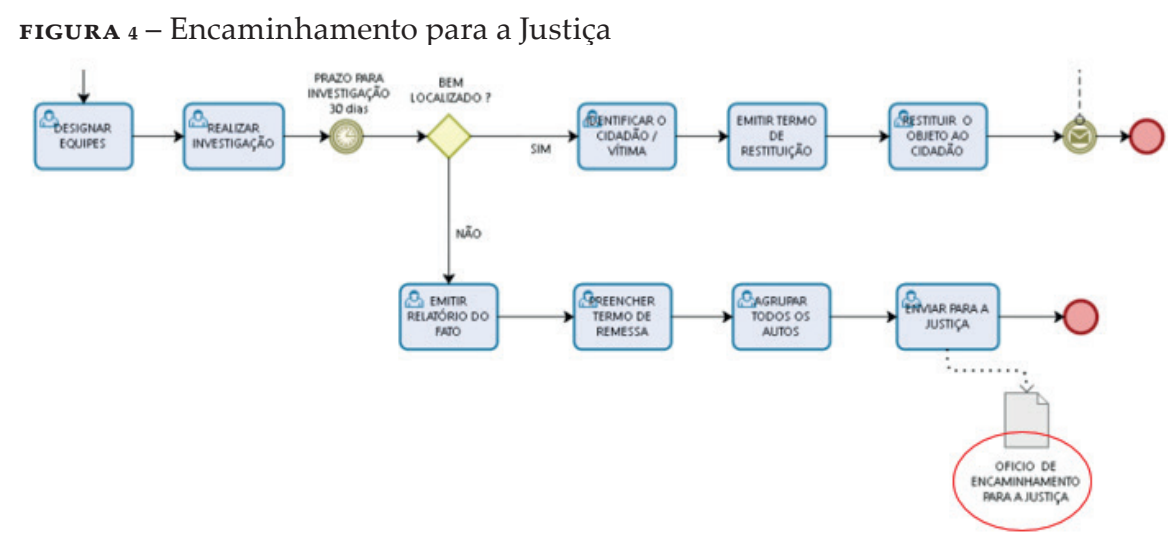

FONTE: Elaborado pelo autor, 2017.

No processo de encaminhamento para a justiça, não foram detectados mecanismos ou tecnologias digitais de rastreabilidade, que pudessem auxiliar na administração destes objetos até os locais onde ficarão custodiados pelo Estado.

\section{PROCEDIMENTOS PARA BENS RECUPERADOS}

No contexto dos bens que são recuperados, a interação dos artefatos com os registros em banco de dados, ocorre primeiramente de modo visual ou por informações contidas fisicamente nestes materiais. A identificação mais precisa e específica dos objetos fica prejudicada em função da ausência de instrumentos, que permitam relações de vínculo e desta forma, tudo que não tenha associação real, é armazenado e remetido ao Poder Judiciário posteriormente.

Para as situações em que ocorre a recuperação dos objetos por intervenção das forças de segurança, na maioria dos casos, o cidadão nem sempre é notificado e os objetos apreendidos acabam sendo remetidos à Justiça e incluídos no Sistema Nacional de Bens Apreendidos - (SNBA). O Registro de 153.343 ocorrências envolvendo terminais celulares roubados/furtados em Minas Gerais no ano de 2016, representaram $33,74 \%$ das ocorrências do REDS, onde apenas $5 \%$ foram recuperados e restituídos às vítimas.

A inclusão de tecnologia poderá ser aplicada a qualquer material independente de sua característica física preservando a integridade do objeto. Para que seja efetivada a restituição, são necessárias de 3 a 4 etapas/procedimentos com um envolvimento mínimo de 3 recursos humanos até que objeto seja devolvido para a vítima conforme Figura 5. 


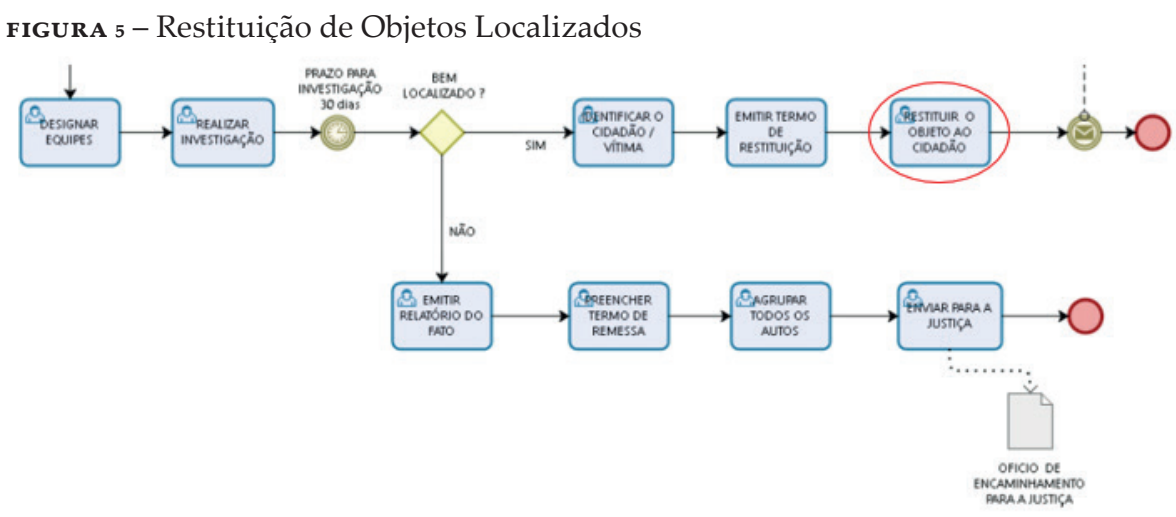

FONTE: Elaborado pelo autor, 2017.

A partir dos recursos da Tecnologia da Informação e Comunicação (TIC), existentes nas etapas que envolvem a localização e restituição de objetos para as vítimas de crimes contra o patrimônio, observa-se que há parâmetros indexadores que podem permitir maior rapidez no processo de localização.

Para todos os materiais que são apreendidos e não há vínculo com as vítimas, os procedimentos de encaminhamento para a Justiça são realizados através de um ofício de remessa. Durante o mapeamento do processo não foram identificados mecanismos tecnológicos que permitissem a identificação rápida e eficiente da origem dos materiais, bem como qualquer associação direta que pudesse apoiar e viabilizar a parametrização rápida com sistemas de informação ou pessoas vinculadas aos bens apreendidos. Os encaminhamentos para a Justiça ocorrem sem a aplicação de tecnologias de rastreabilidade ou sistemas que permitam a administração e um monitoramento futuro adequado.

\section{INTERAÇÃO COM O SISTEMA NACIONAL DE BENS APREENDIDOS}

Para a inclusão dos registros são utilizadas infraestruturas computacionais privadas com acessibilidade através de plataformas web que apresentam diversos níveis de segurança e de controle. Os recursos humanos disponíveis estão associados aos representantes do Estado de MG para assistir o cidadão, simbolizados constitucionalmente pela Secretaria de Segurança Pública e do Poder Judiciário, que estabelecem os parâmetros de gestão, supervisão e coordenação destes sistemas web. Neste cenário, diversas leis e marcos regulatórios disciplinam o funcionamento das instituições que estão contidas neste universo, bem como dá apoio para a prestação dos serviços de segurança pública e de defesa social.

Embora as organizações desenvolvam suas atividades em função de um mesmo propósito, que é garantir a aplicação das leis respeitando as liberdades individuais e o bem-estar social, não é possível afirmar se existe uma forte parametrização entre todos os bancos de dados de registros disponíveis. 
Os sistemas de fiscalização, como os utilizados pelas Receitas Estaduais e Federais, possuem parâmetros de indexação que permitem realizar associações e vínculos, com o objetivo de apoiar o governo em ações de arrecadação mais eficientes. Já outro exemplo de bancos de dados independente pode ser identificado nos procedimentos para o registro da carteira de identidade, que apresenta processos semelhantes em diversos estados da federação, mas que não é convergente para um registro único, ou seja, o modelo existente permite que o indivíduo solicite uma carteira de identidade em cada unidade da federação de maneira independente para o mesmo tipo de registro.

Portanto, diante destes cenários, vê-se também que o sistema nacional de bens apreendidos não possui uma integração mais efetiva com os órgãos de segurança que operacionalmente realizam a apreensão, identificação, registro e a custódia de objetos, seja por extravio, furto, roubo, desvio condicional ou de origem desconhecida. A análise dos questionários permitiu identificar que, na modelagem da etapa de remessa através de ofício, não há uma integração imediata com banco de dados para todos os casos de encaminhamento de objetos furtados/ roubados ou apreendidos.
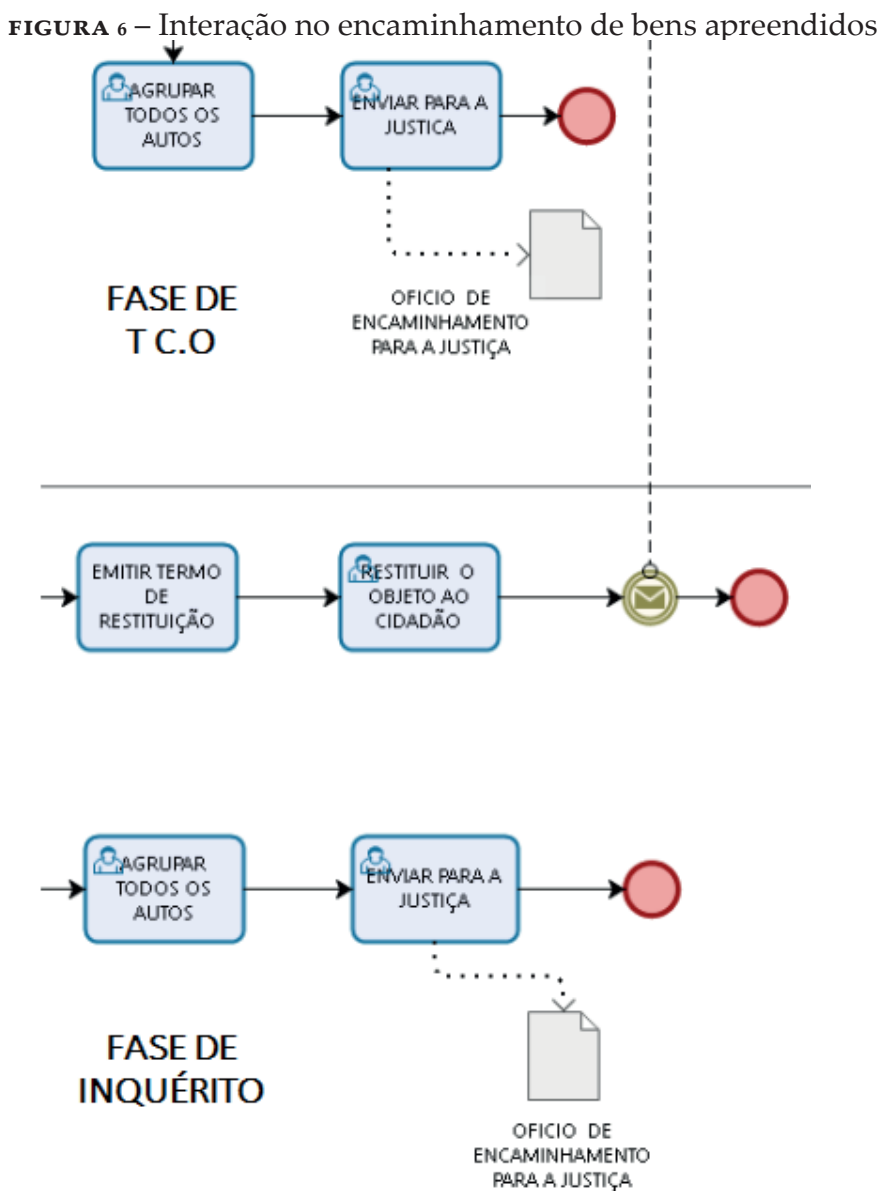

FONTE: Elaborado pelo autor, 2017. 
Tanto para o caso da fase de TCO quanto a fase de Inquérito, os bens são enviados para a Justiça documentalmente por meio de um ofício de remessa conforme verificado na Figura 6. A identificação de um modelo aplicando a modelagem por gestão de processos de negócio, permitiu analisar as relações, desde o início da ocorrência até a restituição do objeto ao cidadão, ou o devido encaminhamento deste bem apreendido ao Poder Judiciário.

FIGURA 7 - Modelo AS-IS completo

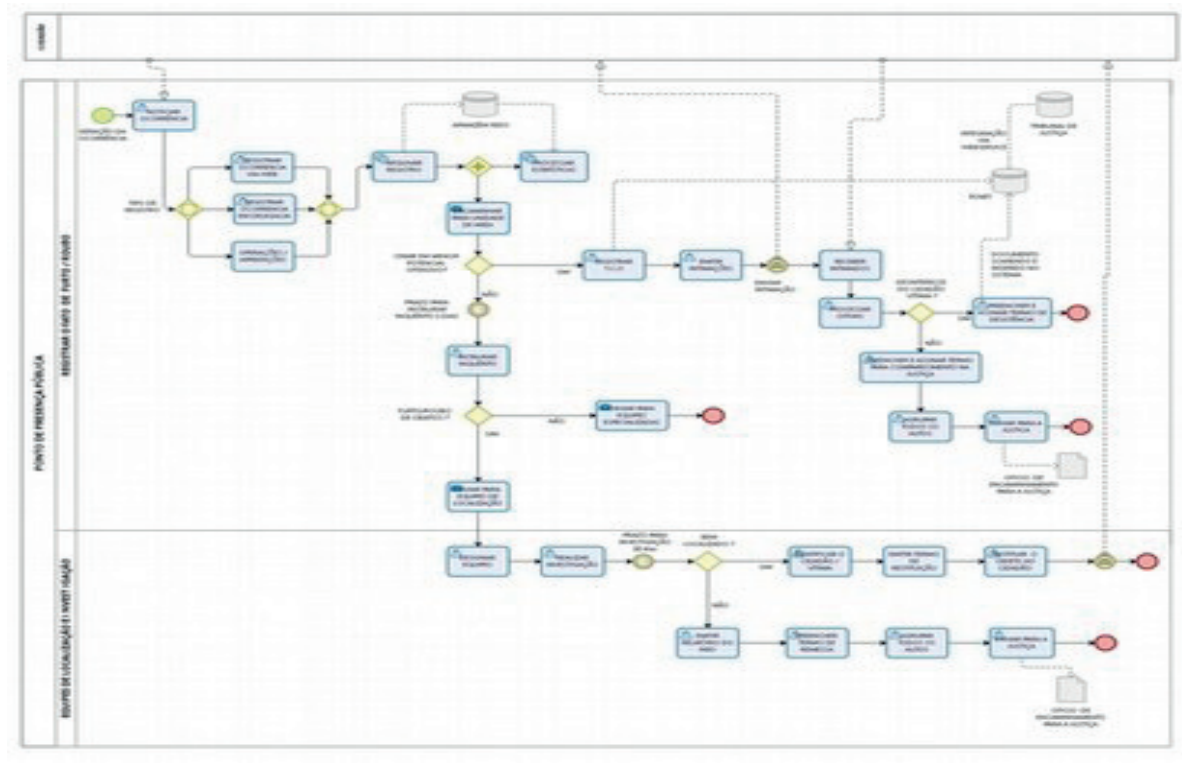

FONTE: Elaborado pelo autor, 2017.

Desta forma, foi possível descrever o modelo baseado em uma visão atual (AS-IS) aplicando técnicas BPMN, para a modelagem dos processos que envolvem o registro das ocorrências e as atividades associadas. A composição final do cenário de interação na gestão e no processamento destas ocorrências foi retratada na Figura 7.

\section{MODELAGEM DO CENÁRIO FUTURO}

Vislumbrando o cenário futuro desejado (TO-BE), a expectativa foi de aumentar a capacidade de resposta em relação à administração dos objetos apreendidos, associados aos registros das ocorrências. Após a análise dos 40 questionários aplicados para o modelamento do cenário atual (AS-IS), no intuito de realizar a validação do modelo, foram realizadas entrevistas semiestruturadas com quatro respondentes: dois funcionários em unidades de Belo Horizonte e dois em unidades de Contagem. Os funcionários selecionados são profissionais com alta qualificação e capacidade técnica de gestão, supervisão e coordenação nas áreas envolvidas em cada unidade.

TCO - Registro de Ocorrências para crimes de menor potencial ofensivo.
Foi modelado o TO-BE que buscou melhorar a aplicação dos recursos, inserir tecnologia e diminuir o tempo de resposta de interação entre a finalização das ocorrências do Poder Judiciário. As etapas que 
Revista de Gestão Pública

PRATICAS E DESAFIOS

ISSN: 2177-1243

Webservice: interface de integração entre sistemas que permite enviar e receber dados. anteriormente eram executadas conforme demonstrado na Figura 5, passarão a ser realizadas com um suporte administrativo de monitoramento de gestão, retratado na Figura 8.

A incorporação de tecnologias de rastreabilidade, além de facilitar a localização, ainda permitirão uma maior eficiência nos procedimentos de registro, restituição e remessa para a Justiça, também identificado na Figura 8. A incorporação da tecnologia poderá ser ofertada pelo Estado ou pela iniciativa privada no sentido de garantir ou preservar a aquisição de objetos de alto valor agregado pelo cidadão.

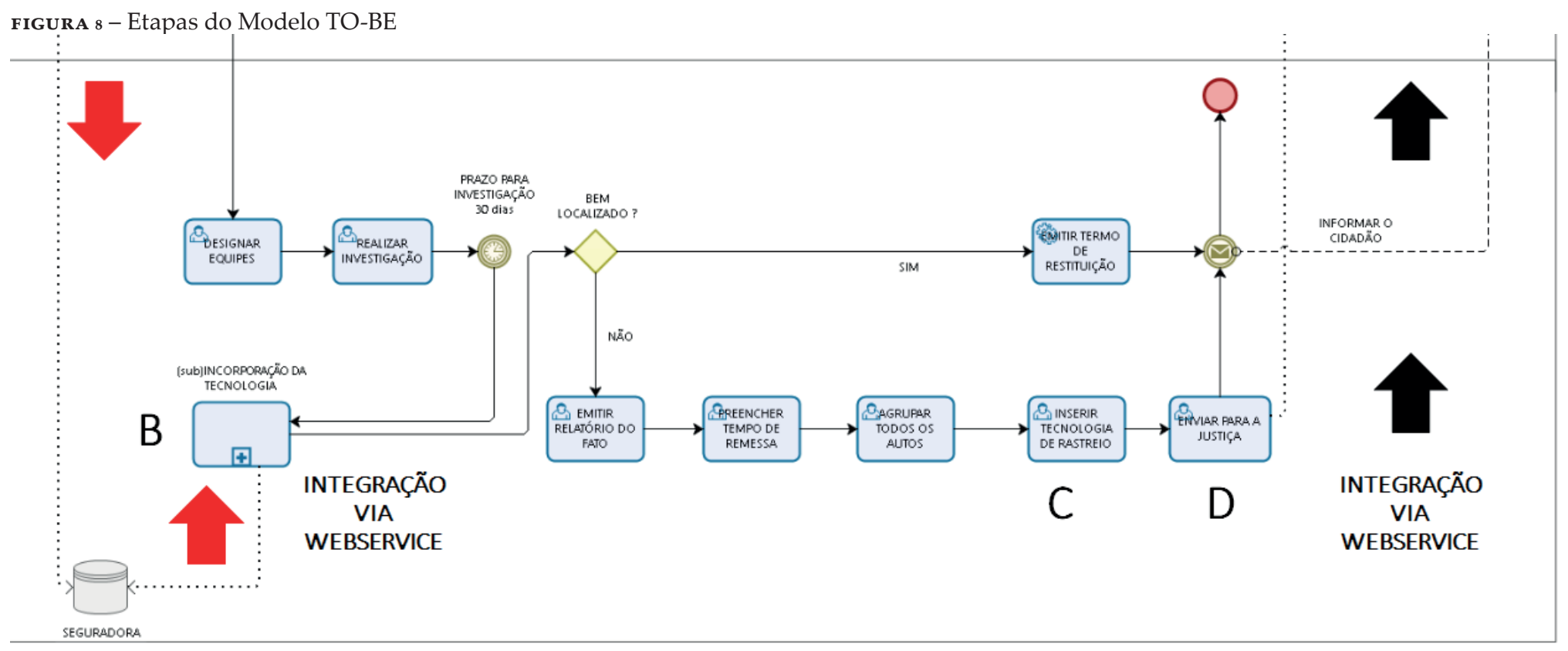

FONTE: Elaborado pelo autor, 2017

A proposta do novo modelo sugere que haja uma associação antecipada e dinâmica, entre a etapa de registro da ocorrência e sistemas informação ou banco de dados externos. A integração de novos serviços via webservice poderá aumentar a interação e, desta forma, diminuir o tempo de resposta para a localização de um objeto que tenha tecnologia de rastreabilidade incorporada conforme identificado nos pontos A e B da Figura 9. Caso o objeto não seja localizado, um 
mecanismo de rastreabilidade poderá ser incorporado com o posterior encaminhamento para a justiça com um lastro tecnológico e sistêmico, demonstrado nas etapas C e D da Figura 9.

FIGURA 9 - Modelagem TO-BE - Interação e Tecnologia

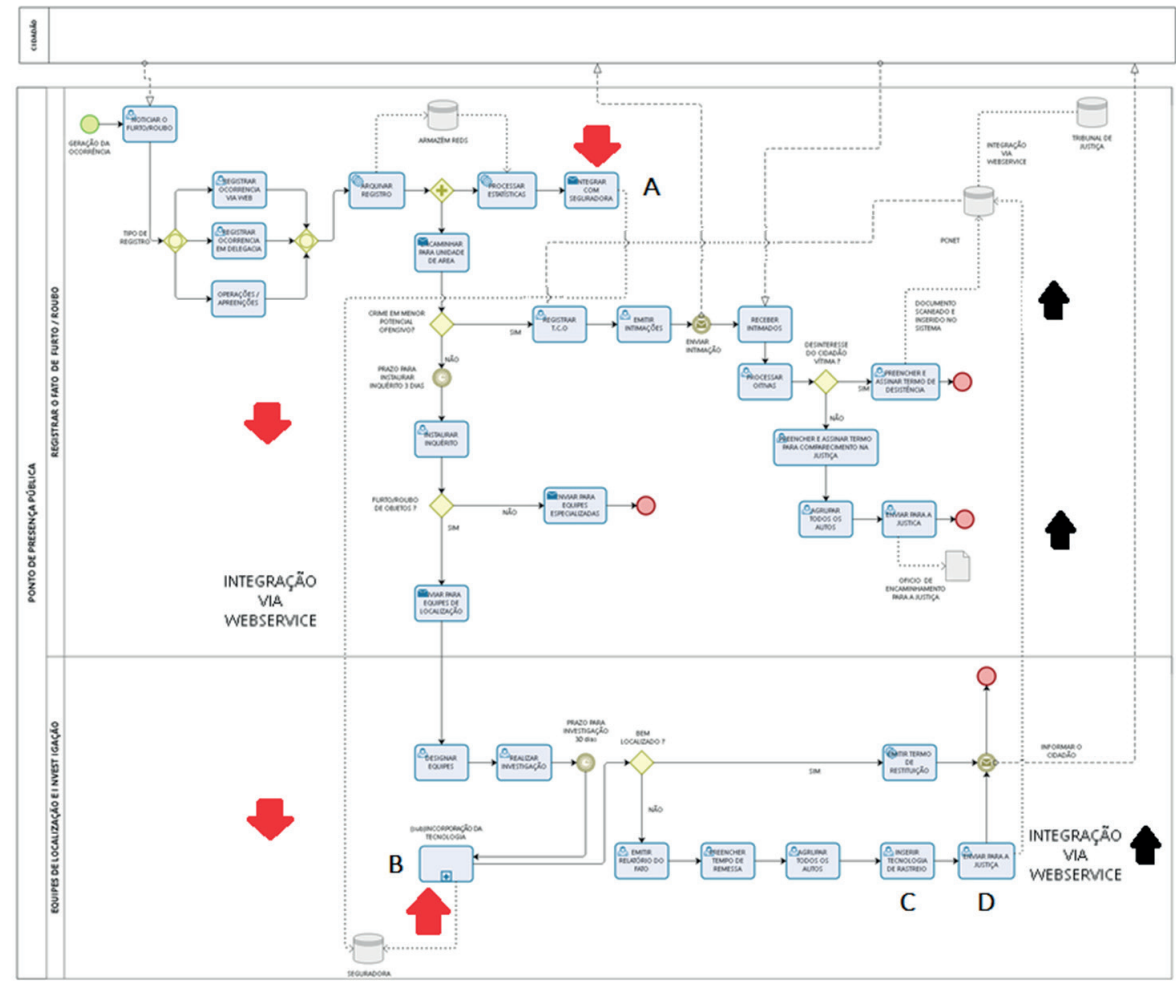

FONTE: Elaborado pelo autor, 2017.

Considerando a análise dos recursos humanos associados aos processos AS-IS e TO-BE, verificou-se que a inclusão de tecnologia e a exclusão de intervenções humanas também poderá aumentar a velocidade dos processos. Para que seja possível melhorar a capacidade de uso destes recursos, é conveniente um estudo quantitativo mais profundo, por meio do desenvolvimento de pesquisas voltadas à simulação dos processos identificados.

\section{CONSIDERAÇÕES FINAIS}

No contexto atual, a aplicação da notação BPMN permitiu modelar e identificar de maneira sistêmica, todas as etapas e atividades desde a geração do registro da ocorrência até sua finalização. O processo de validação destacou que a aplicação de mecanismos, sugerindo tecnologias de rastreabilidade, mostrou-se possível a diminuição do tempo de resposta para as ações necessárias referentes a restituição de bens apreendidos.

Foi possível, também, verificar a dimensão da tecnologia e de recursos humanos, físicos e de infraestrutura, necessários para a implantação de projetos que tornem mais eficientes as atividades desen- 
volvidas pela segurança pública e pelo poder judiciário.

O objetivo central do trabalho foi alcançado, pois permitiu identificar os aspectos mais importantes que poderão ser aplicados para reprimir o avanço da criminalidade, por meio da gestão e da tecnologia. Os processos foram mapeados de modo que foi possível estabelecer as relações mais importantes, otimizar e melhorar a interação e a capacidade de resposta das instituições na administração e restituição de bens apreendidos.

A abordagem permitiu visualizar cenários em que, futuramente, será importante uma atenção em relação às expectativas do cidadão quanto à disponibilidade de novos serviços, assim como o aumento da credibilidade nas ações das forças de segurança.

A sinalização de investimentos associados ao estabelecimento de políticas públicas, através do desenvolvimento de projetos, inclusive envolvendo a iniciativa privada, também são importantes para a criação de novos serviços de interesse do Estado. Desta forma, para que seja possível envolver os ambientes relacionados, esta pesquisa idealizou cenários e discussões para sensibilizar as necessidades em processos de gestão e de inovação dentro dos segmentos públicos e privados.

Uma das propostas de estudos futuros é a implantação de tecnologias de rastreabilidade que permitam realizar ou prever uma geolocalização aproximada de objetos a serem monitorados. O estabelecimento de parcerias privadas poderia contribuir com produtos e serviços integrados com a segurança pública, para preservar ou segurar um bem patrimonial. A roteirização permitirá entender por causas aleatórias de deslocamentos, previsões dos padrões de comportamento de pessoas e dos objetos associadas ao longo do tempo (BLOCH; CORREIA; PALAVRAS, 2014). Desta forma, seria possível antecipar a localização aproximada de objetos e pessoas, aplicando um modelo de Programação Matemática Linear, que permitirá uma ação sobre o indivíduo que esteja portando um determinado artefato.

\section{REFERÊNCIAS}

ABPMP. Abpmp. Journal of Chemical Information and Modeling v. 53, n. 9, p. 1689-1699, 2013.

BLOCK, S., Correia, A. R., \& Palavras, R. (2014). Aplicação de Programação Linear em um problema de roteirização com Rastreabilidade do roteiro, Conferencia: XVII Simpósio de Pesquisa Operacional e Logística da Marinha (2), 1-11.

BRAGA, Lamartine Vieira, et al. O papel do Governo Eletrônico no fortalecimento da governança do setor público. Revista do Serviço Público v. 59, n. 1, p. 5-21, 2008. 
Universidade do Vale do Itajaí - UNIVALI, 2008.

CARRIJO, Carmen Vânia; ALVARENGA, Lúcio Ismael de. Qualidade do Atendimento Prestado ao Cliente-Cidadão da Secretaria Municipal de Regulação Urbana de Aparecida de Goiânia: Um Estudo Exploratório e Propositivo. Convibra, 2011. Disponível em: $<\mathrm{http}$ ://www.convibra.com. br/dwp.asp?id=3104\&ev=23>. Acesso em: o9 mar. 2017.

COMPLOIER, Mylene. Gestão e Destinação dos Bens Apreendidos no Processo Penal.17ıf., Dissertação - Universidade Presbiteriana Mackenzie, 2015.

COUTINHO, Luciano, et al. Parcerias públicoprivadas no brasil - Revista FGV Projetos, 2014.

CRITSINELIS, Marco Falcão. Confisco Criminal. p. 1-146 , 2013. Disponível em: <http://www.ajuferjes.org.br/ PDF/confiscocriminal.pdf $>$. Acesso em: 21 fev. 2017

FREY, Klaus. Governança eletrônica: experiências de cidades europeias e algumas lições para países em desenvolvimento. Internet e Política teoria e prática da democracia eletrônica 48f. - Conferência Eletrônica do Centro Virtual de Estudos Políticos (CEVEP), 2000.

JUSTIÇA, Conselho Nacional de. Manual de bens apreendidos, 2011.

LIMA, Anne Cristiny. $\mathbf{O}$ confisco de ativos e bens nos delitos de lavagem de dinheiro. p. 1-106, Dissertação - Faculdade de Ciências Jurídicas da Universidade Tuiuti do Paraná, 2012.

LUI, Márcio de la Cruz; OLIVEIRA, Bráulio. Posicionamento Estratégicos de Produtos com Inovação. RAI - Revista de Administração e Inovação p 53-69, 2015.

MA, X. Rfid-based business process and workflow management in healthcare: design and implementation, 2012. Disponível em: <http://digitalcommons.wayne. ed u/oa_dissertations/551/>. Acesso em: 21 fev. 2017.

MEDEIROS, Paulo Henrique Ramos; GUIMARÃES, Tomás de Aquino. Contribuições do governo eletrônico para a reforma administrativa e a governança no Brasil. Revista do Serviço Público v. 56, n. 4, p. 449-464, 2005.

MEDEIROS, Paulo Henrique Ramos; GUIMARÃES, Tomás de Aquino. A Relação Entre Governo Eletrônico e Governança Eletrônica no Governo Federal Brasileiro. v. III, p. 16 , 2004. Disponível em: <http://www.anpad. org.br/ diversos/trabalhos/EnAPG/enapg_2004/2004_ 
ENAPG96.pdf>.1679-3951. Acesso em: 03 mar. 2017.

MODRAK, Vladimir; KNUTH, Peter. Architecture Design and Implementation of RFID Based Academic Library

.Research Journal of Applied Sciences. [S.l: s.n.], 2012.

NACIONAL, Conselho. Relatório Trimestral da Ouvidoria.

CNJ Conselho Nacional de Justiça. Jan a mar. de 2016.

OLIVEIRA, Uanderson Rébula de, et al. Logistica Reversa e Identificação de Produtos: Revisão Teórica para Industria Eletroeletrônica. Revista Produção Online, p. 633-677, 2016.

PEDROSO, Marcelo C.; ZWICKER, Ronaldo; SOUZA, Cesar A. Adoção de RFID no Brasil: um estudo exploratório. Revista de Administração p. 12-36, 2009. Disponível em: <http://www. scielo.br/pdf/ram/vioni/vioniao2.pdf $>$. Acesso em: 21 fev. 2017.

ROBERTO, Cláudio et al. Gestão Estratégica do Conhecimento em Produção: Uso da Tecnologia RFID (RadioFrequency Identification) para Controle de Equipamentos Ativos em Ambientes Industriais. p. 2092-2114 - 1oth International Conference on Information Systems and Technology Management - CONTECSI , 2013.

SAADI, Ricardo Andrade; MACHADO, Diogo de Oliveira. Recuperação de ativos desviados por corrupção : soluções inovadoras para lidar com a criminalidade econômica. Revista de Doutrina e Jurisprudência, p. 32-51, 2015.

SANTOS, Higor Monteiro; SANTANA, André Felipe; ALVES, Carina Frota. Análise de Fatores Críticos de Sucesso Da Gestão De Processos De Negócio Em Organizações Públicas. Revista Eletrônica de Sistemas de Informação v. 11, n. 1, p. 1-21 , 2012. Disponível em: <http://www.periodicosibepes.org.br/ojs/ index.php/reinfo/article/ view/996>. Acesso em: 13 dez. 2016.

SILVA, Eugénia Maria Vicente da. Fatores relevantes na satisfação global do cidadão no relacionamento com os serviços públicos municipais : Estudo de caso da Câmara Municipal de Sabrosa. p.1-14, 2012. Disponível em: <http:// repositorium. sdum.uminho.pt/handle /1822/20762>. Acesso em: 21 fev. 2017.

TINOCO, Denise Hernandes Berger A. S.; CHAHINE, Marien Abou. A psicologia e o novo paradigma da ciência. Dissertação - Universidade de São Paulo p. 105-121, 2012. 